\title{
Widening Participation in Medicine: The Impact of Medical Student-Led Conferences for Year 12 Pupils
}

\author{
Ben Ryan (iD) \\ Charlotte Auty $\mathbb{D D}^{2}$ \\ Matthew Maden (ID $^{3}$ \\ Amy Leggett (iD ${ }^{4}$ \\ Alisha Staley (D) $^{3}$ \\ Enam Haque (1D ${ }^{3}$
}

'Department of Medicine, Lancashire Teaching Hospitals NHS Foundation

Trust, Preston, Lancashire, UK; ${ }^{2}$ Surgical

Division, Queen's Medical Centre,

Nottingham University Hospitals NHS

Trust, Nottingham, Nottinghamshire, UK;

${ }^{3}$ University of Manchester Medical

School, The University of Manchester,

Manchester, Greater Manchester, UK;

${ }^{4}$ Department of Undergraduate

Education, East Lancashire Hospitals NHS

Trust, Blackburn, Lancashire, UK
Correspondence: Ben Ryan

Department of Medicine, Lancashire

Teaching Hospitals NHS Foundation

Trust, Preston, Lancashire, United

Kingdom

Tel +44I772 7I6565

Email benryan@doctors.org.uk
Purpose: Individuals from lower socio-economic backgrounds are under-represented in the medical profession: confidence is a barrier to them successfully applying to medical school. This study examined the impact of two student-led conferences for Year 12 pupils, at which they had the opportunity to present their work. It looked at the ability of the conferences to improve participant confidence, and the feasibility of its replication by other student-led groups.

Methods: The first, Conference A, had more time and finances invested into it than the second, Conference B. The latter relied solely on university society funding, but utilised WP criteria for selection of participants. Participants identified their confidence in six areas on a ten-point scale, immediately before and after the intervention.

Results: A paired $t$-test showed a significant improvement $(p<0.01)$ in all areas of confidence for both conferences. Cohen's $d$ showed Conference A had larger effect sizes in five out of six areas than Conference B.

Conclusion: This intervention has demonstrated a significant positive impact on participant confidence: a key factor to improve their chance of successful admission to medical school. Supporting participants with their presentations prior to the conference was found to further enhance their confidence. The authors feel that this work could be replicated successfully by other student groups.

Keywords: widening access, social mobility, medical school admissions, confidence

\section{Introduction}

The most under-represented group in medicine are those from lower socioeconomic backgrounds. ${ }^{1}$ Widening participation (WP) aims to improve access to higher education for under-represented groups by providing support and opportunity. ${ }^{2}$ Data from The Medical Schools Council Selection Alliance revealed that only $14 \%$ of new medical students in 2015 were from lower socio-economic groups. ${ }^{3}$ This is striking given that these groups represent $56 \%$ of the United Kingdom population. ${ }^{4}$ A 4-fold increase in the number of WP students is therefore required to ensure that doctors are representative of the community they serve.

Lower socio-economic areas struggle to recruit doctors. ${ }^{5}$ Medical students from lower socio-economic backgrounds often decide to work in these areas after graduating. ${ }^{6}$ Widening participation to medicine may therefore improve health inequalities by addressing this recruitment issue. Literature suggests that pupils from lower socio-economic backgrounds are less likely to believe that they can study medicine, making them less likely to apply. ${ }^{7}$ This may be because they do not 
see themselves represented in the field, because they have no medical role models, and because of a lack in confidence in their abilities. ${ }^{8,9}$

WP initiatives give young people exposure to people from similar backgrounds who have overcome similar barriers to gain a place at medical school. ${ }^{10}$ This provides medical role models to improve self-confidence and may result in more WP pupils successfully applying to medicine. ${ }^{11}$ In 2016, the Medical Schools Council proposed several initiatives to increase the amount of outreach work undertaken by medical schools. One proposal included holding regional conferences for pupils aspiring to study medicine. Privately operated events like this can cost pupils more than $£ 300$ to attend, making it inaccessible to pupils from lower socio-economic backgrounds. ${ }^{12}$

Funding for WP outreach work is a limitation for medical schools. Interviews with Deans at 25 medical schools in the United Kingdom highlighted that finances and staffing availability were a significant problem in the commissioning of WP work. $^{13}$ The authors proposed a student-led approach to WP work, to overcome barriers such as resource costs and staff time. Medical students can successfully engage in 80 hours of WP work over the course of an academic year, alongside studying their fulltime degree. ${ }^{14}$ They can also deliver large-scale events. ${ }^{15}$

This paper aims to identify if a WP initiative can improve pupil confidence in applying to medicine, which can then be replicated internationally. The authors were part of Manchester Outreach Medics, a university medical WP society. They discussed their student-led work in a recent article. ${ }^{16}$ Their previous work helped in improving pupils' understanding in key areas but did not address pupil confidence. They created and delivered two conferences, in 2017 and 2018, at which Year 12 pupils delivered their own presentations. To the authors' knowledge, this style of intervention was unique and has not been previously discussed in the literature.

The primary aim of this paper was to evaluate the impact of this conference on participant confidence. The paper also explores the feasibility of other student-led groups replicating this intervention, and the robustness of WP participant selection for both conferences.

\section{Materials and Methods}

\section{Intervention Delivery}

The first conference (Conference A) occurred in 2017, with funding of $£ 4000$ provided by The University of
Manchester, Office for Fair Access and National Collaborative Outreach Programme. It was organised by BR (Ben Ryan) as part of a 12-week project in the University of Manchester Medical School programme. The itinerary for Conference A is included as supplemen tary data. It was a whole day event, beginning with lectures and a panel discussion on "life in healthcare". Pupils could then choose to attend from a menu of workshops, including research, compassion in medicine, medical ethics, professionalism, public health, and holistic medicine. All participants delivered their own presentations to a group of their peers and a panel of medical student volunteers. Participants selected and researched a topic of their choosing and delivered a presentation during the conference. BR provided academic and pastoral support to pupils through this process, helping them to decide on a topic, identifying valuable resources, and providing feedback on their presentations.

The second conference, Conference B, was organised by CA (Charlotte Auty) in 2018. It was similar to Conference A, but adapted for CA to deliver this alongside her full-time medical studies. In contrast to Conference A, CA was not able to support each participant with the preparation of their presentation. The budget was $£ 800$ and provided by The University of Manchester and Students' Union. The initial lecture and panel discussion were the same, but the workshops differed slightly: interview skills, empathy in medicine, medical ethics, professionalism, treating the patient as a person, and holistic medicine.

\section{Participant Recruitment}

The authors invited sixth form pupils in Greater Manchester and Lancashire to the conferences. Greater Manchester was felt to be an appropriate area for WP activities, as Manchester has one of the highest proportions of deprived neighbourhoods on the English Indices of Deprivation. ${ }^{17}$ Lancashire is a cold spot for medical WP work, with low levels of pupils admitted to medical school. ${ }^{12}$

The two conferences differed in their recruitment of pupils. Conference A identified high-priority sixth forms using the Manchester Prioritisation Model (MPM). This tool identifies schools and sixth forms under-represented in higher education. ${ }^{18}$ High-priority sixth forms in Greater Manchester were invited first, followed by other sixth forms in Lancashire and Greater Manchester. 81 pupils attended Conference A, all delivering their own 
Table I Demographics Used as WP Flags

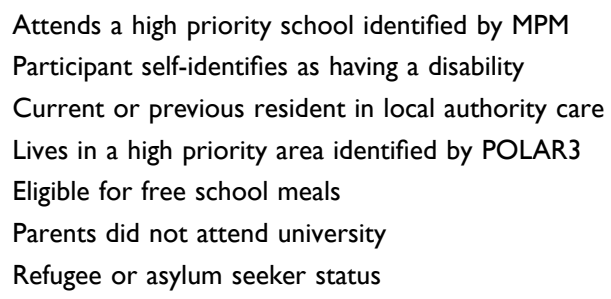

Abbreviations: WP, Widening Participation; MPM, Manchester Prioritisation Model; POLAR3, Participation of Local Areas classification groups.

presentations. Conference B had a more robust approach to pupil recruitment, using WP flags (Table 1). A WP flag is a particular demographic criterion which is underrepresented in the medical profession.

Pupils applied for Conference B through a secure, encrypted data collection platform named Bristol Online Surveys. All those who applied with at least one WP flag were automatically granted attendance. 104 pupils attended, with 48 delivering their own presentations.

\section{Data Gathering}

Participants were asked to complete an anonymised preconference ten-point questionnaire on arrival, highlighting their level of confidence in different areas. Zero equated to no confidence; ten equated to complete confidence. This questionnaire, including the areas assessed, is shown in Table 2. Participants were asked to complete the questionnaire again immediately at the end of the conferences. The authors paired each participant's pre- and post-conference questionnaires together, to allow for paired analysis of the data.

Manchester Outreach Medics use a ten-point questionnaire for all their events, and this questionnaire was in a similar format. This enables the team to compare their interventions. Furthermore, other student-led WP groups could feasibly create similar questionnaires to allow for comparison between different groups and their interventions.

\section{Data Analysis}

The authors calculated the mean confidence levels for each of the areas for both conferences. The authors discounted data for an area if a participant did not include both preand post-conference answers on that area. The methodology used was consistent with the Declaration of Helsinki and participants provided informed consent to take part in this study. The authors used the University of Manchester's Ethics Decision Tool and The National Health Service Health Research Authority's Ethics Decision tool to confirm that ethical approval was not needed for this study.

They used a paired $t$-test to assess for a statistically significant difference in mean confidence levels between the pre- and post-conference data $(p<0.05)$. Cohen's $d$ then enabled them to calculate an effect size on the confidence levels. Effect sizes of greater than 0.8, 0.5, and 0.2 were considered large, medium, and small, respectively. ${ }^{19}$ Effect sizes between Conference A and Conference B were compared, to assess if there was any difference between the impact of the conferences on participant confidence.

\section{Results}

76 participants, out of the 81 who attended Conference A, completed both questionnaires. There was a statistically significant increase in confidence for all areas tested: two areas demonstrated a medium effect size in improvement; four areas demonstrated a large effect size. The most relevant areas for medical school applicants were overall

Table 2 Participant Questionnaire

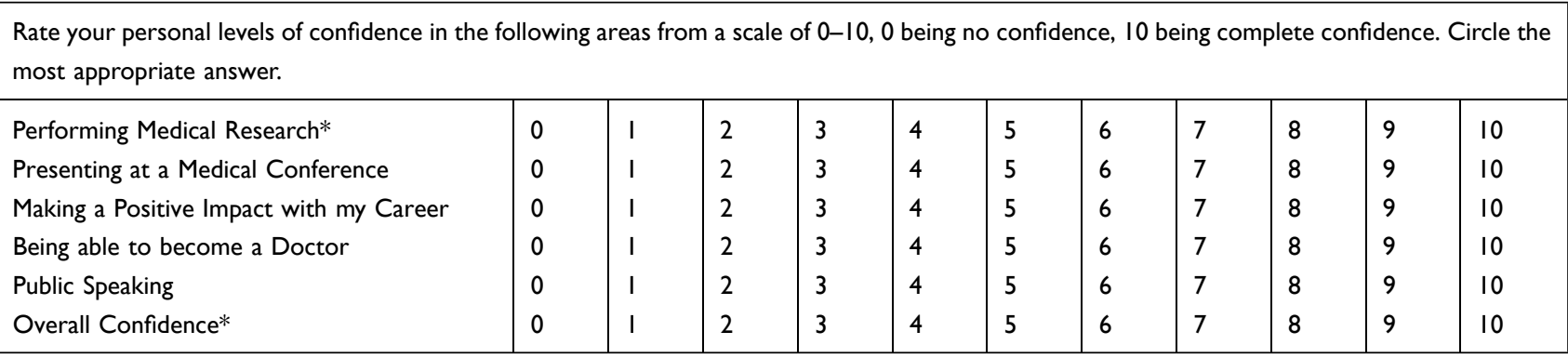

Notes: *In these areas on the pre-conference questionnaire, participants were asked to estimate their level of confidence before they obtained a ticket for the conference. This was done as it was expected that the support participants received from the medical student organisers prior to the conference would have affected their confidence in these areas. 
confidence (Increase, 1.75; CI, 1.26-2.24; Effect Size, $0.90 ; p<0.01$ ) and being able to become a doctor (Increase, 1.41; CI, 0.92-1.90; Effect Size, 0.71; $p<0.01$ ).

96 participants, out of the 104 who attended Conference B, completed both questionnaires. There was a statistically significant increase in confidence levels for all areas, with one area showing a small effect size, one area showing a medium effect size, and four areas showing a large effect size. The most relevant areas for medical school applicants were overall confidence (Increase, 1.50; CI, 1.02-1.98; Effect Size, 0.82; $p<0.01$ ) and being able to become a doctor (Increase, 0.75; CI, 0.24-1.26, Effect Size, 0.41, $p<0.01$ ). Comparing the two conferences, Conference A had larger effect sizes than Conference $B$ in five out of six areas. The results of both Conference $\mathrm{A}$ and $\mathrm{B}$ are displayed in Table 3.

\section{Discussion}

The aim of this study was to assess what impact the student-led conferences had on pupil confidence. Confidence is a crucial factor for medical school applicants and should be addressed by WP initiatives. ${ }^{7-9}$ Both conferences showed a statistically significant increase in levels of confidence in all the areas tested. Conference A showed a greater effect size and improvement in confidence than Conference $\mathrm{B}$ in five out of six areas. The authors speculate that this may be due to BR having more time to mentor participants prior to Conference A, than CA did for Conference B. This is consistent with Vygotsky's Zone of Proximal Development, ${ }^{20}$ as the pupils improved their confidence further when receiving support from the medical student organiser.

Both conferences engaged young people in Lancashire, a cold spot area for medical outreach work. ${ }^{12}$ However, they utilised different methods for participant recruitment. Conference A did not use individual participant data to determine admission to the event. As a result, it is possible that many of the pupils at Conference A were not the appropriate target audience for WP initiatives. For Conference B, the encrypted application system used meant that potential participants could provide data on WP flags securely. As a result, all applicants with at least one WP flag were provided admission to attend.

The authors concluded that Conference B was more successful at targeting the appropriate audience for WP initiatives. It also had a higher attendance level, which the authors feel is due to more sixth forms knowing about Manchester Outreach Medics' work and reputation. A good reputation and adhering to strict WP criteria for attendance are therefore good pointers for student groups wishing to replicate this work.

Table 3 Confidence Data for Both Conference A and Conference B

\begin{tabular}{|c|c|c|c|c|c|c|c|c|c|}
\hline \multirow[t]{2}{*}{ Area } & \multirow[t]{2}{*}{ A/B } & \multirow[t]{2}{*}{ n } & \multicolumn{2}{|c|}{$\begin{array}{l}\text { Pre- } \\
\text { Conference }\end{array}$} & \multicolumn{2}{|c|}{$\begin{array}{l}\text { Post- } \\
\text { Conference }\end{array}$} & \multirow[t]{2}{*}{$\begin{array}{l}\text { Increase in Mean } \\
(95 \% \mathrm{Cl})\end{array}$} & \multirow[t]{2}{*}{ Effect Size } & \multirow[t]{2}{*}{$p$-value } \\
\hline & & & Mean & SD & Mean & SD & & & \\
\hline \multirow[t]{2}{*}{ Performing Medical Research } & A & 74 & 5.32 & 2.32 & 7.11 & 2.44 & $1.78(1.21-2.36)$ & 0.75 & $<0.01$ \\
\hline & B & 95 & 4.68 & 2.45 & 6.81 & 2.47 & $2.13(1.44-2.8 I)$ & 0.87 & $<0.01$ \\
\hline \multirow[t]{2}{*}{ Presenting at a Medical Conference } & $A$ & 75 & 5.45 & 2.44 & 8.41 & 1.61 & $2.96(2.38-3.54)$ & 1.43 & $<0.01$ \\
\hline & B & 96 & 4.73 & 2.47 & 6.92 & 2.67 & $2.19(1.42-2.95)$ & 0.85 & $<0.01$ \\
\hline \multirow[t]{2}{*}{ Making a Positive Impact with my Career } & $A$ & 74 & 6.53 & 2.10 & 8.64 & 1.21 & $2.11(1.67-2.55)$ & 1.23 & $<0.01$ \\
\hline & B & 96 & 6.88 & 1.98 & 8.40 & 1.50 & $1.52(1.00-2.04)$ & 0.87 & $<0.01$ \\
\hline \multirow[t]{2}{*}{ Being Able to Become a Doctor } & A & 75 & 6.59 & 2.01 & 7.99 & 1.95 & $1.41(0.92-1.90)$ & 0.71 & $<0.01$ \\
\hline & B & 96 & 6.91 & 1.95 & 7.66 & 1.73 & $0.75(0.24-1.26)$ & $0.4 I$ & $<0.01$ \\
\hline \multirow[t]{2}{*}{ Public Speaking } & $A$ & 75 & 6.13 & 2.41 & 8.17 & 1.85 & $2.04(1.50-2.58)$ & 0.95 & $<0.01$ \\
\hline & B & 96 & 6.55 & 2.14 & 8.14 & 1.85 & $1.58(1.05-2.11)$ & 0.79 & $<0.01$ \\
\hline \multirow[t]{2}{*}{ Overall Confidence } & $A$ & 75 & 6.41 & 2.09 & 8.16 & 1.77 & $1.75(1.26-2.24)$ & 0.90 & $<0.01$ \\
\hline & B & 96 & 6.71 & 1.91 & 8.21 & 1.73 & $1.50(1.02-1.98)$ & 0.82 & $<0.01$ \\
\hline
\end{tabular}

Notes: Effect size $=$ Cohen's $d$ effect size, which equates to increase in mean divided by pooled standard deviation: $0.2 \leq$ small effect size; $0.5 \leq$ medium effect size; $0.8 \leq$ large effect size. $p$-value = result of the paired $t$-test: the probability of the change in mean confidence being due to chance. Data is displayed to two decimal places. Abbreviations: $n$, Number of questionnaires with both pre- and post-conference data completed; A, Conference A Data; B, Conference B Data; SD, Standard Deviation; $\mathrm{Cl}$, Confidence Interval. 
Time is also essential for those wishing to run a similar intervention. CA organised Conference $\mathrm{B}$ alongside her regular studies, illustrating that it is feasible for conferences of this magnitude to be organised by full-time medical students. Students considering a similar conference also need to consider funding. Funding for Conference B, for 104 participants, was $£ 800$. The authors obtained this through university society funding which is feasible for other student groups to achieve. The largest expense for both conferences was catering. This implies that overhead costs could be reduced by not having catering for the conference.

The aims from this study have been met, and its findings are continuing to have impact on the authors' WP work: MM delivered a similar conference in 2019 and AS is adapting the conference to be delivered following COVID-19 restrictions; BR and AL are adapting the participant presentation activity to a different WP project working with the same target audience. The authors hope that WP groups will replicate or adapt this work. It is a feasible intervention that can target WP pupils and significantly improve their confidence in applying to medical school

\section{Limitations}

This study had several limitations. It did not assess for long-term impact measures, such as confidence levels later in the year or percentage of participants who successfully gained a place in medical school. This would have provided meaningful evidence of sustainable impact of the conferences. Furthermore, confidence is more complex than a written scale, which may not accurately reflect improvements in confidence. Participants may have also felt inclined to augment their scores on the postconference questionnaire, possibly out of gratitude to the organisers. Future studies should consider qualitative evaluation with focus groups, to explore the experience of the pupils.

The organisers of both Conference A and B had previously delivered many large events for the same target audience. Through this, they developed relevant skills, a team of dedicated medical student volunteers, a reputation with schools in their target area, and a supportive network at the University of Manchester. These factors likely contributed to the success of the conferences. Other medical student groups may not have this expertise, support, or experience to replicate the success of the conferences.
The conferences took place prior to the COVID-19 pandemic, which has affected how WP activities are delivered. Manchester Outreach Medics have adapted their events to deliver them online. It is unknown how this will affect the impact of their work. A potential benefit is that the events can reach further areas without participants having to travel. This reduces the financial impact for young people from disadvantaged backgrounds. A possible limitation is that participants would need technology and good quality internet connection, which may put WP pupils at a disadvantage. Manchester Outreach Medics plan to gather data to compare the efficacy of the online events to their in-person events.

\section{Conclusion}

This study has shown that medical student-led conferences, at which Year 12 pupils deliver their own presentations, significantly improve pupil confidence. Supporting pupils prior to the conference with their presentations further improves confidence. This study emphasises the importance of using WP flags for recruitment, to ensure the conference is truly catering for WP pupils. Finally, it has shown that it is feasible for full-time medical students to deliver these conferences with minimal funding. WP groups would be welcome to replicate this work, and to contact the corresponding author for further information or advice.

Recommendations for future research are to evaluate the impact of this intervention using qualitative data such as focus groups, and to evaluate the effect on applications and admissions to medical school. The Higher Education Access Tracker could facilitate this, as it allows WP initiatives to track participants as they progress through their studies. ${ }^{21}$

\section{Abbreviations}

WP, Widening Participation; BR, Ben Ryan; CA, Charlotte Auty; MPM, Manchester Prioritisation Model; POLAR3, Participation of Local Areas classification groups; SD, Standard Deviation; CI, Confidence Interval; MM, Matthew Maden; AS, Alisha Staley; AL, Amy Leggett.

\section{Data Sharing Statement}

The data that supports the findings of this study is available from the corresponding author upon request.

\section{Ethics Approval and Informed Consent}

The methodology used was consistent with the Declaration of Helsinki and participants provided informed consent to 
take part in this study. The University of Manchester's Ethics Decision Tool and The National Health Service Health Research Authority's Ethics Decision tool were used to confirm that ethical approval was not needed for this study.

\section{Acknowledgments}

The authors would like to thank and acknowledge Katie Barnes for supporting the organisation and supporting BR and $\mathrm{CA}$ in leading the team. The authors thank and acknowledge Helen Franklin for her support of the organisation. The authors thank and acknowledge those who ran individual sessions and panellists: James Adams, Lily Edwards, Holly Gibson, Alfred So, Ameera Selant, Dr Luke Cannon, Dr Clare Rayner, Dr Sunil Aggarwal, and Professor Madhavi Paladugu. The authors thank and acknowledge all volunteers in Manchester Outreach Medics for their hard work in delivering the conferences. The authors thank and acknowledge the General Medical Council and Medical Schools Council for their support with the conferences, particularly their representatives who delivered sessions: Ioanna Maraki and Clare Owen, respectively. The authors thank and acknowledge David McNally for his support and for delivering a lecture at Conference B.

\section{Author Contributions}

BR designed and organised Conference A. BR acquired data for Conference A. BR delivered a session at Conference B. BR contributed to the abstract, material and methods, results, and discussion. BR revised the introduction and the conclusion. CA designed and organised Conference B. CA assisted in the delivery of Conference A. CA contributed to the material and methods, results, discussion, and conclusion. CA performed the data analysis for this study. MM assisted in the delivery of Conference B. MM contributed to the interpretation of the data. MM searched for, and reviewed, relevant literature and contributed to the introduction. MM revised the material and methods, and discussion. AL assisted in the delivery of Conference A. AL assisted in the conception of Conference A and the study. AL reviewed relevant literature and contributed to the introduction. AL revised the material and methods, results, and discussion. AS assisted in the delivery of Conference B. AS contributed to the interpretation of the data. AS reviewed relevant literature and contributed to the background. AS revised the material and methods, and discussion. EH supervised and contributed towards the conception of Conference A and the study. EH contributed to session delivery at Conference A. EH revised the abstract, introduction, material and methods, results, discussion, and conclusion. $\mathrm{BR}, \mathrm{CA}, \mathrm{MM}, \mathrm{AL}$ and AS met regularly to design the study, interpret the data, and revise the manuscript. All authors made substantial contributions to conception and design, acquisition of data, or analysis and interpretation of data; took part in drafting the article or revising it critically for important intellectual content; agreed to submit to the current journal; gave final approval of the version to be published; and agree to be accountable for all aspects of the work.

\section{Funding}

The authors thank and acknowledge the University of Manchester's Faculty of Biology, Medicine and Health and Students' Union, Office for Fair Access, and National Collaborative Outreach Programme for providing funding to deliver these conferences. The authors thank and acknowledge the University of Manchester's Faculty of Biology, Medicine and Health Widening Participation team for providing funding to publish this study.

\section{Disclosure}

BR was assessed as part of his medical degree from The University of Manchester for his work on Conference A, for which $\mathrm{EH}$ was his supervisor. The authors report no conflicts of interest in this work.

\section{References}

1. Medical Schools Council Selection Alliance. Indicators of Good Practice in Contextual Admissions. London; 2018. Available from https://www.medschools.ac.uk/news/selection-alliance-publishesguidance-on-contextual-admissions. Accessed March 16, 2021.

2. Apampa A, Kubacki A, Ojha U, Xiang J. Challenges in widening participation outreach: is enough being done to tackle the under-representation of low-income students in medicine? Adv Med Educ Pract. 2019;10:917-923. doi:10.2147/AMEP.S211895

3. Medical Schools Council Selection Alliance. Selection Alliance 2017 Report: An Update on the Medical Schools Council's Work in Selection and Widening Participation. London; 2017. Available from https:// www.medschools.ac.uk/our-work/selection/selecting-for-excellence. Accessed March 16, 2021.

4. Office of National Statistics. NS-SeC. Nomis; 2011. Available from: https://www.nomisweb.co.uk/census/2011/qs607ew. Accessed March 16, 2021.

5. Hutt P, Gilmour S. Tackling Inequalities in General Practice. The King's Fund. London; 2010. Available from https://www.kingsfund. org.uk/projects/gp-inquiry/health-inequalities. Accessed March 16, 2021.

6. Dowell J, Norbury M, Steven K, Guthrie B. Widening access to medicine may improve general practitioner recruitment in deprived and rural communities: survey of GP origins and current place of work. BMC Med Educ. 2015;15:1. doi:10.1186/s12909-015-0445-8 
7. Ball R, Alexander K, Cleland J. "The biggest barrier was my own self": the role of social comparison in non-traditional students' journey to medicine. Perspect Med Educ. 2020;9(3):147-156. doi:10.1007/s40037-020-00580-6

8. McHarg J, Mattick K, Knight LV. Why people apply to medical school: implications for widening participation activities. Med Educ. 2007;41(8):815-821. doi:10.1111/j.1365-2923.2007.02798.x

9. Mathers J, Parry J. Why are there so few working-class applicants to medical schools? Learning from the success stories. Med Educ. 2009;43(3):219-228. doi:10.1111/j.1365-2923.2008.03274.x

10. Nicholson S, Cleland JA. "It's making contacts": notions of social capital and implications for widening access to medical education. Adv Health Sci Educ Theory Pract. 2017;22(2):477-490. doi:10.1007/s10459-016-9735-0

11. Kamali AW, Nicholson S, Wood DF. A model for widening access into medicine and dentistry: the SAMDA-BL project. Med Educ. 2005;39(9):918-925. doi:10.1111/j.1365-2929.2005.02227.x

12. Medical Schools Council. Implementing Selecting for Excellence: A Progress Update. London; 2016. Available from https://www.med schools.ac.uk/our-work/selection/selecting-for-excellence. Accessed March 16, 2021.

13. Cleland JA, Nicholson S, Kelly N, Moffat M. Taking context seriously: explaining widening access policy enactments in UK medical schools. Med Educ. 2015;49(1):25-35. doi:10.1111/medu.12502
14. Azmy J, Nimmons D. Reflections on a widening participation teaching role. Clin Teach. 2017;14(2):139-140. doi:10.1111/tct.12574

15. Ojha U, Patel S. Student-led widening access schemes. Adv Med Educ Pract. 2017;8:581-585. doi:10.2147/AMEP.S139836

16. Ryan B, Kitchen A, Chan A, Gibson H, Haque E. Widening participation to medicine: a student-led workshop for medical school applicants. MedEdPublish. 2018;7:2. doi:10.15694/mep.2018.0000130.1

17. Ministry of Housing Communities and Local Government. The English Indices of Deprivation 2019 (Iod2019). London; 2019. Available from https://www.gov.uk/government/statistics/englishindices-of-deprivation-2019. Accessed March 16, 2021.

18. The University of Manchester. Widening Participation Strategic Assessment. Manchester; 2009. Available from https://documents.man chester.ac.uk/display.aspx?DocID=8305. Accessed March 16, 2021.

19. Cohen J. Statistical Power Analysis for the Behavioural Sciences. 2nd ed. New York: Lawrence Erlbaum Associates; 1988.

20. Vygotsky LS. Mind in society: development of higher psychological processes. In: Cole M, Jolm-Steiner V, Scribner S, Souberman E eds. Cambridge, MA; London, England: Harvard University Press; 85-86:1978.

21. Higher Education Access Tracker. Welcome to HEAT; 2020. Available from: https://heat.ac.uk/. Accessed March 21, 2021.
Advances in Medical Education and Practice

\section{Publish your work in this journal}

Advances in Medical Education and Practice is an international, peerreviewed, open access journal that aims to present and publish research on Medical Education covering medical, dental, nursing and allied health care professional education. The journal covers undergraduate education, postgraduate training and continuing medical education
Dovepress

including emerging trends and innovative models linking education, research, and health care services. The manuscript management system is completely online and includes a very quick and fair peer-review system. Visit http://www.dovepress.com/testimonials.php to read real quotes from published authors. 